\title{
The upgrade of the T2K Near Detector ND280
}

\author{
John Nugent ${ }^{* \dagger}$ \\ University of Glasgow \\ E-mail: john.nugenteglasgow.ac.uk
}

The T2K-II program will upgrade the beam power of the main ring at J-PARC to $1.3 \mathrm{MW}$ and will also renovate the $\mathrm{T} 2 \mathrm{~K}$ near detector ND280. With these two components the $\mathrm{T} 2 \mathrm{~K}$ collaboration will establish the value of leptonic $\mathrm{CP}$ violation at the $3 \sigma$ level for a significant fraction of the possible $\delta_{C P}$ values. An upgrade of the near detector is essential to achieving this goal as it can be used to reduce the overall statistical and systematic uncertainties to the level of less than $4 \%$. We have developed an innovative concept for this neutrino detection system, comprising the totally active Super-Fine-Grained-Detector (SuperFGD), two High Angle TPC (HA-TPC) instrumented with resistive Micromegas and six TOF planes. The SuperFGD, a highly segmented scintillator detector, acting as a fully active target for the neutrino interactions, is a novel device [1]. We have demonstrated that this detector, providing three 2D projections, has excellent PID, timing and tracking performance, including a $4 \pi$ angular acceptance, especially important for short proton and pion tracks. The HA-TPC will be used for 3D track reconstruction, momentum measurement and particle identification. In the summer of 2018 we tested prototypes of the SuperFGD, the resistive Micromegas and the ToF in a CERN PS test beam with excellent results. We have recently completed a detailed TDR describing all the components of the ND280 Upgrade [1] and the project has been recently approved by CERN as part of the Neutrino Platform (NP07).

The 21st international workshop on neutrinos from accelerators (NuFact2019)

August 26 - August 31, 2019

Daegu, Korea

\footnotetext{
* Speaker.

${ }^{\dagger}$ on behalf of the T2K collaboration
} 


\section{T2K Experiment}

The J-PARC facility produces an intense $2.5^{\circ}$ off-axis beam of $v_{\mu}$ where $\sim 1 \%$ of the flux is $v_{e}$ and the neutrino flux peaks at $0.6 \mathrm{GeV}$. The beam line runs in both $\nu \& \bar{v}$ mode with the neutrino energy spectrum and flavour content measured first at the J-PARC site with ND280, the near detector, and then again with the Super-Kamiokande detector which is located $295 \mathrm{~km}$ away. The main goal of the T2K experiment is to measure the oscillation of $v_{\mu}$ to $v_{e}$ and the phase of CP violation in neutrino oscillations $\left(\delta_{C P}\right)$.

\section{Current Near Detector - ND280}
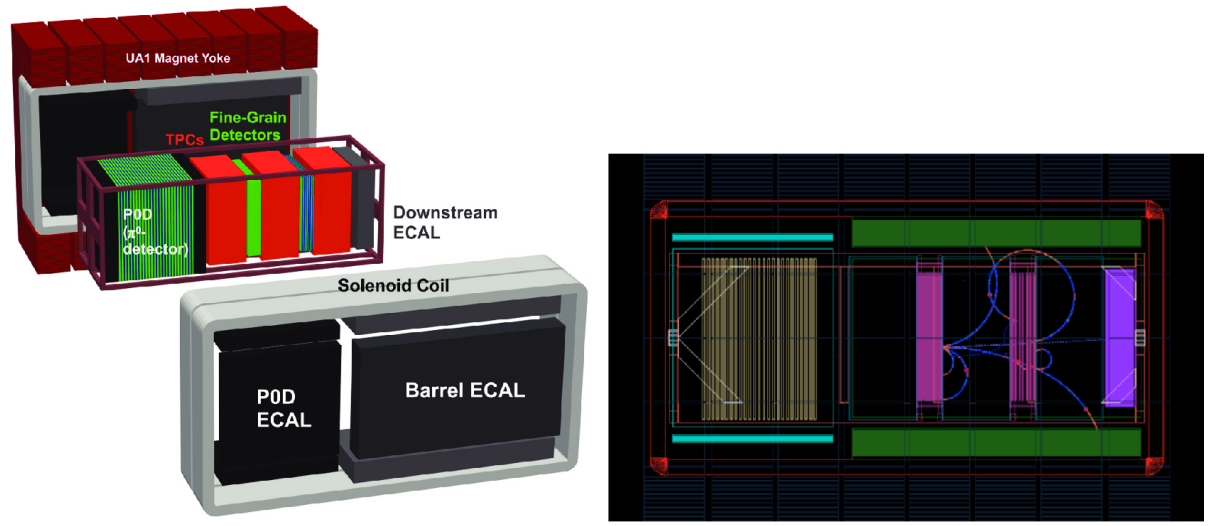

Figure 1: Schematic of current ND280 (left) and event display from ND280 (right).

The current near detector is shown in figure 1. It consists of the UA1 magnets which provide a 0.2 T magnetic field allowing for charge identification, 2 Fine-Grained detectors (FGD) composed of planes of scintillator bars along the $X Y$ direction (perpendicular to the neutrino beam) [2], 3 Time Projection Chambers (TPC) [3], the Ecal which reconstructs $\gamma \& e$ [4], the Side-Muon Range Detector (Side-MRD) which tracks $\mu$ [5] and the P0D which consists of bars of scintillator/lead foil and measures $\pi^{0}$ production [6].

\section{Physics Goals of ND280 Upgrade}

The main goal of the upgrade is to reduce the systematic errors on the flux and cross-section measurements necessary for the oscillation analysis. In order to achieve this it is necessary to improve the models describing nuclear effects in neutrino-nucleus interactions including charged current multi-nucleon interactions (CCMN) and understand the effects of hadronic final state interactions (FSI) which can modify the multiplicity and kinematics of secondaries created by neutrinonucleus interactions. To minimise the uncertainties in the extrapolation from the near to far detector of the constraints on such nuclear effects it is also desirable to match the acceptance of the near detector to that of Super-Kamiokande the far detector. 
A lower momentum threshold for final state particle detection (not only muons and pions but also protons) and $4 \pi$ coverage of the fully-active interaction volume increases the sensitivity to observables like the transverse variables [7] which allows the nuclear effects to be characterised with better precision.

\section{Upgrade Proposal}

For the upgraded near detector a similar performance in term of momentum resolution, $\mathrm{dE} / \mathrm{dx}$ and charge measurement to that currently provided by ND280 is required. Other specifications include a fiducial mass of a few tons and highly efficient $4 \pi$ tracking for low energy $\pi \mathrm{s}$ and protons to determine event topology, with proton-pion identification. Furthermore, good timing determination (at the $0.5 \mathrm{~ns}$ level) that can be used to distinguish inward going background events from outward going neutrino interactions is required. A schematic of the upgraded ND280 detector is shown in figure 2 .

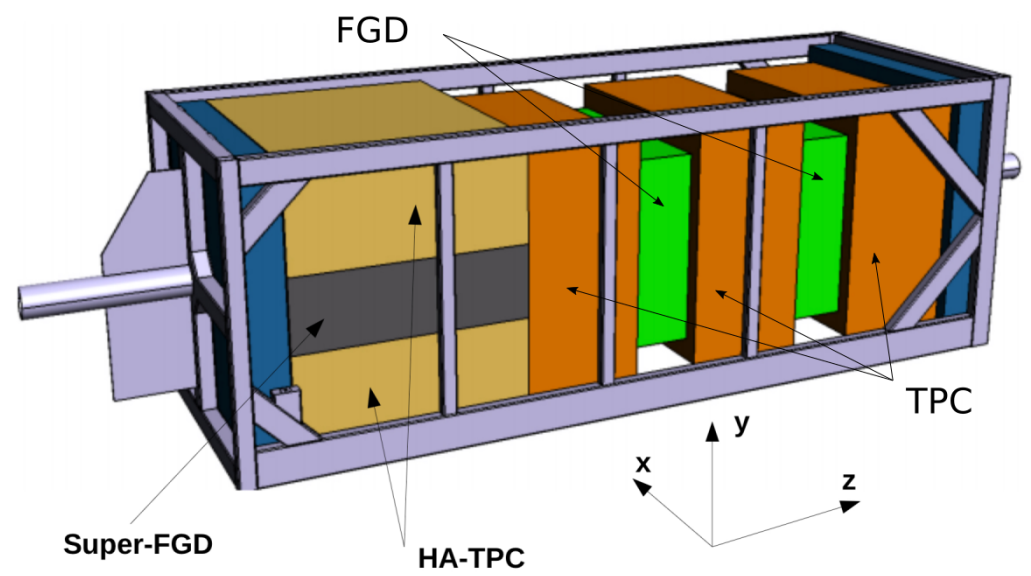

Figure 2: A schematic of the ND280 upgrade [1].

The SuperFGD (figure 3) is a novel idea for a fine grained fully-active plastic scintillator detector made of many optically independent $1 \mathrm{~cm}$ cubes. The scintillator is a composite of polystyrene doped with $1.5 \%$ paraterphenyl and covered by $\sim 50 \mu \mathrm{m}$ of chemical reflector consisting of polystyrene doped with $\mathrm{TiO}_{2}$. Each cube has three holes of $1.5 \mathrm{~mm}$ diameter drilled along their $\mathrm{X}, \mathrm{Y}$ and $\mathrm{Z}$ axes through which WLS fibres are fed. The full detector is $1.8 \times 2.0 \times 0.6$ $m$ comprising 2,064,384 cubes and readout through 58,368 channels. It has twice the resolution of the existing FGD and provides 3D reconstruction. A prototype was built in the summer of 2018 with a test beam conducted at the CERN PS T9 area. The purpose of this exercise was to test the assembly, readout, electronics and preliminary data analysis of the SuperFGD prototype.

The High Angle TPCs (HA-TPC) will be $1.8 \times 0.7 \times 2.12 \mathrm{~m}$ with a composite field cage and instrumented with resistive Micromegas. They will have a gas composition of $95 \% \mathrm{Ar}, 3 \% \mathrm{CF}_{4}$ and $2 \% \mathrm{iC}_{4} \mathrm{H}_{10}$ consistent with the current TPCs. It will provide a space point resolution of $\sim 300 \mu \mathrm{m}$ and a $\Delta p / p$ of $10 \%$ at $1 \mathrm{GeV} / \mathrm{c}$. The similarity of the design to the current T2K TPCs should allow for straightforward construction and operation. The Mircomegas resistive layer in the HA-TPCs spreads charge predictably across pixels for better track resolution. 

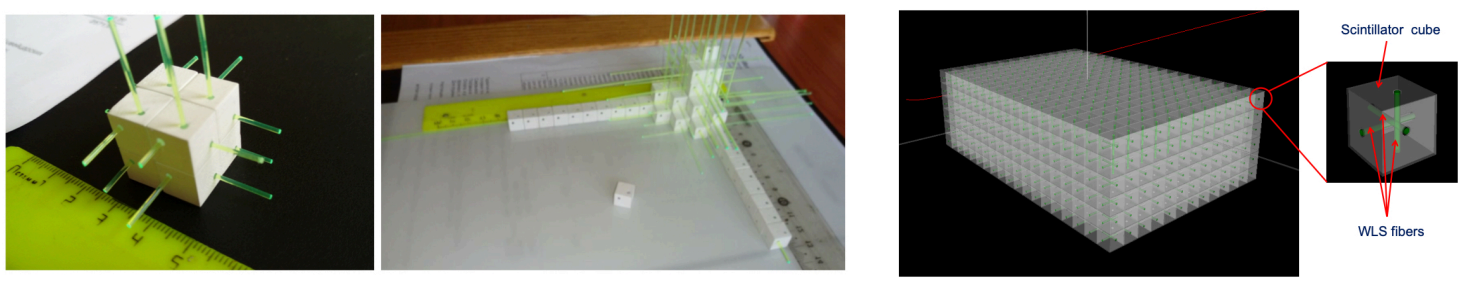

Figure 3: The scintillator cubes which compose the SuperFGD are shown in this figure. Arranged first in smaller units of $4 \times 4$ (left) building up to the complete SuperFGD (second right, schematic) [1].

\section{Upgraded Sensitivity}
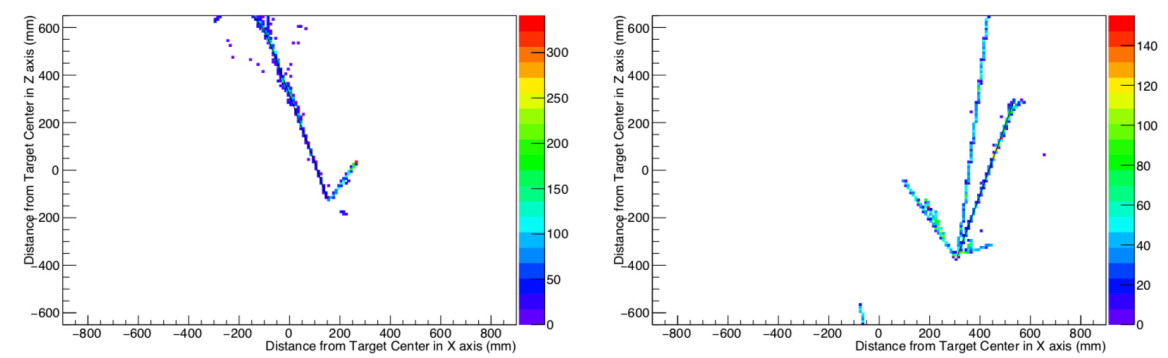

Figure 4: Event display from SuperFGD [1].
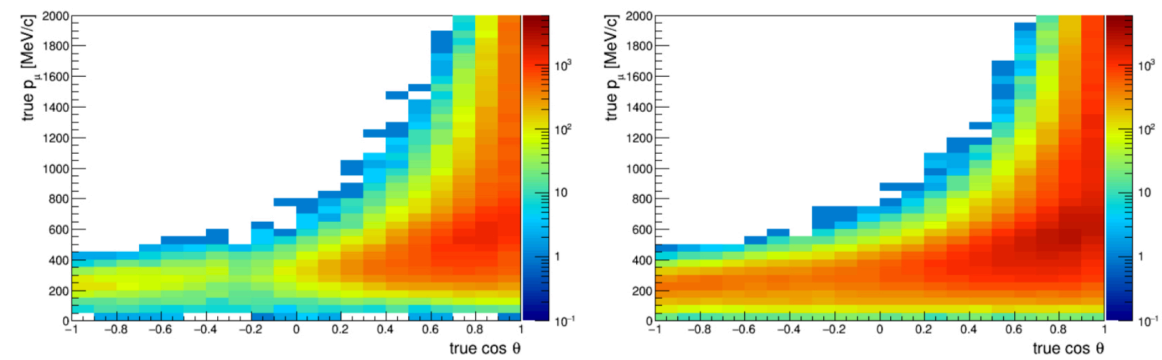

Figure 5: Left: Acceptance of current ND280. Right: Acceptance of upgraded ND280 [1].

The high granularity of the SuperFGD allows for improved pattern recognition and track reconstruction (figure 4) compared to the current ND280. Furthermore the improved acceptance, see figure 5, in the high angle region matches Super-Kamiokande. The enhanced ability to reconstruct low momentum tracks allows the nuclear models describing neutrino-nucleus interactions to be tuned with greater precision. These results only include events where muons reaching the TPCs, even better results have been demonstrated including tracks reconstructed with the SuperFGD alone. These capabilities were tested with a fitter that is functionally identical to the fitter used in the T2K oscillation analysis. The results of this study are shown in figure 6 which demonstrates improved constraints for all flux and cross-section model parameters. The study was done with $8 \times 10^{21}$ POT which is about a third of the expected total data. The final uncertainty on the 
total number of events at SK is reduced by $20-30 \%$ with respect to the same number of POT with the previous ND280 detector.
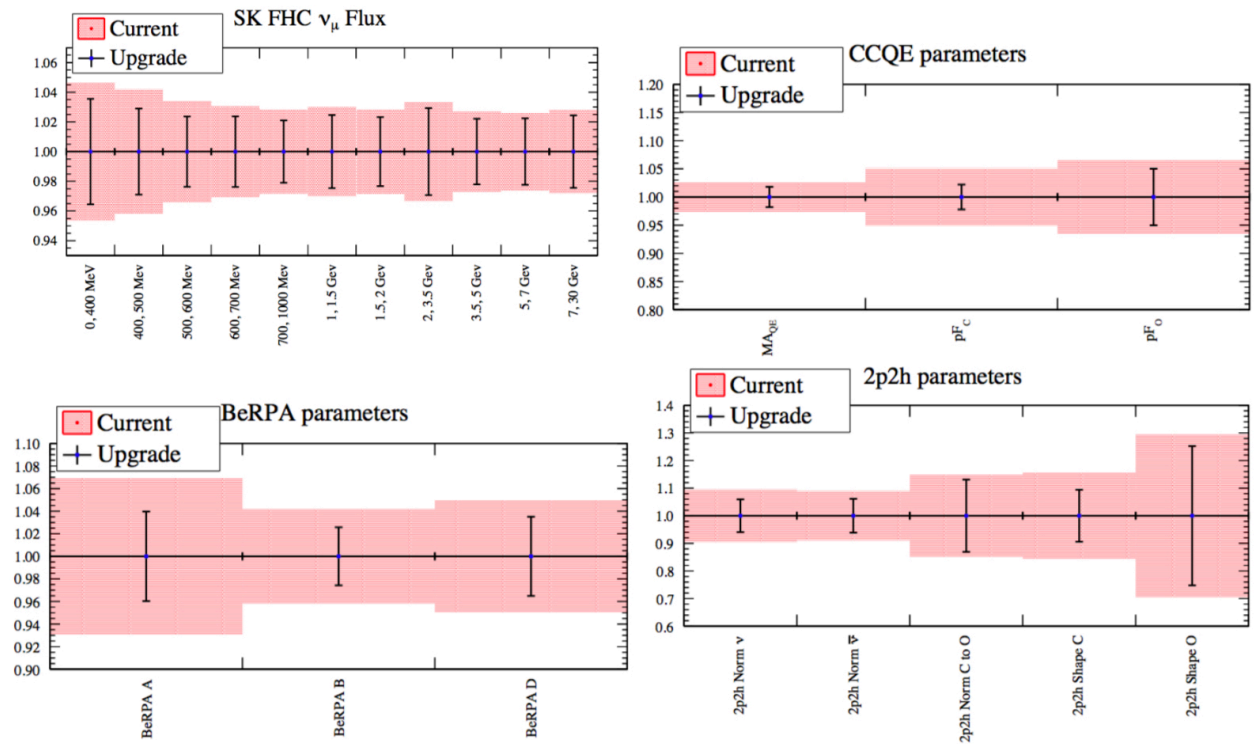

Figure 6: The post-fit errors on the main systematic parameters are shown for both the ND280 upgrade (blue dots) and the current ND280 configuration (red bars). These sets of parameters comprise the far detector muon neutrino flux (top left), the CCQE cross-section (top right), the Random Phase Approximation (bottom left) and $2 \mathrm{p} 2 \mathrm{~h}$ parameters (bottom right). The upgrade reduces systematics by $\sim 30 \%$ [1].

\section{Conclusions}

The T2K phase II program will include an upgrade of the J-PARC main accelerator ring to a beam power of 1.3 MW and an upgrade of the near detector, ND280. Given the dominant effect of neutrino-nucleus interactions in determining the systematics of the oscillation analysis enhancing the capabilities of the near detector is essential. By improving the high angle acceptance and low momentum track reconstruction with SuperFGD, the HA-TPCs and the ToF counters studies have shown that the uncertainty on the oscillation analysis can be better constrained. In preparation for the upgrade the prototype detectors were built and successfully commissioned in the summer of 2018.

\section{References}

[1] T2K Collaboration, K. Abe et al., "T2K ND280 Upgrade - Technical Design Report," arXiv:1901.03750 [physics.ins-det].

[2] T2K ND280 FGD Collaboration, P. A. Amaudruz et al., "The T2K Fine-Grained Detectors," Nucl. Instrum. Meth. A696 (2012) 1-31, arXiv: 1204.3666 [physics.ins-det] .

[3] T2K ND280 TPC Collaboration, N. Abgrall et al., "Time Projection Chambers for the T2K Near Detectors," Nucl. Instrum. Meth. A637 (2011) 25-46, arXiv: 1012.0865

[physics.ins-det]. 
[4] T2K UK Collaboration, D. Allan et al., "The Electromagnetic Calorimeter for the T2K Near Detector ND280," JINST 8 (2013) P10019, arXiv:1308.3445 [physics.ins-det] .

[5] S. Aoki et al., "The T2K Side Muon Range Detector (SMRD)," Nucl. Instrum. Meth. A698 (2013) 135-146, arXiv:1206.3553 [physics.ins-det].

[6] S. Assylbekov et al., "The T2K ND280 Off-Axis Pi-Zero Detector," Nucl. Instrum. Meth. A686 (2012) 48-63, arXiv:1111.5030 [physics.ins-det].

[7] T2K Collaboration, K. Abe et al., "Characterization of nuclear effects in muon-neutrino scattering on hydrocarbon with a measurement of final-state kinematics and correlations in charged-current pionless interactions at T2K," Phys. Rev. D98 no. 3, (2018) 032003, arXiv: 1802.05078 [hep-ex] . 